\title{
Der Erwerb von Verbzweitsätzen mit Subjekt im Mittelfeld bei italophonen DaF-Studierenden. Erwerbsphasen, Lernschwierigkeiten und didaktische Implikationen
}

\author{
Sabrina Ballestracci (Pisa)
}

\begin{abstract}
This paper focuses on the acquisition of German sentences by Italian native speakers in Foreign Language Teaching, with the verb located in second position, and the subject found in the middle field. The study is based upon a corpus of texts written by Italian students during their first six semesters at the University of Pisa. The first part of the study describes the main grammatical structural differences between Italian and German declarative sentences, referring to position of verb, subject and clause constituents. In the second part, I summarize the research results of the main German-Italian linguistic contrastive studies on the acquisition of word order in German, by focusing on declarative sentences with the subject in the middle field. The final part of this paper focuses on the linguistic and contextual factors influencing the acquisition process for further development in this field of study, in order to offer suggestions for foreign language teaching of German.
\end{abstract}

\section{$1 \quad$ Einleitung}

Wie die meisten Studien zum L2-Erwerb des Deutschen bestätigen ${ }^{1}$, bereiten die Bearbeitung und Verinnerlichung einiger Zielstrukturen den L2-Lernern besondere Schwierigkeiten. Diese kommen vor allem beim Erwerb bestimmter grammatischer Regeln bzw. Strukturen der deutschen Sprache vor, die keine Entsprechung in der L1 finden ${ }^{2}$. Nach den Studien, die diese Hypothese, nämlich die kontrastive, akzeptieren, ließe sich das Phänomen besonders in jenen grammatischen Bereichen beobachten, die grundsätzliche Unterschiede zwischen dem Ausgangs- und dem Zielsystem aufweisen.

Unter den verschiedenen Phänomenen, die aus kontrastiver Perspektive relevant für das Sprachenpaar Deutsch-Italienisch sind, werden hier dem Ziel der vorliegenden Arbeit gemäß drei Punkte behandelt: die Verbstellung, die Verwendung des Subjekts und die Satzglied-

\footnotetext{
${ }^{1}$ Der Erwerb des Deutschen als Zweit- bzw. Fremdsprache ist Gegenstand einer relativ großen Menge von Untersuchungen der letzten zehn Jahre, die sich mit unterschiedlichen grammatischen Bereichen beschäftigen (Tschirner 1999, Diehl et al. 2000, Klein Gunnewiek 2000, Thomoglou 2004, Menzel 2004). Mit besonderem Bezug auf den Erwerb des Deutschen bei italophonen Lernern vgl. Clahsen et al. (1983), Crespi Günter (1989), Pienemann (1998, letzte Fassung 2005), Terrasi Haufe (2004) und Ballestracci (2006).

2 Dass den Lernschwierigkeiten Interferenzen aus der L1 zugrunde lägen, wird durch eine beträchtliche Menge von Untersuchungen im DaF- und DaZ-Bereich mehr oder weniger indirekt bestätigt (Clahsen et al. 1983, Crespi-Günther 1989, Tschirner 1999; Diehl et al. 2000, Terrassi-Haufe 2004, Ballestracci 2006). Allerdings stellt die kontrastive Hypothese noch eine umstrittene Frage dar, zumal einige Autoren behaupten, dass die zwischen L1 und L2 bestehenden strukturellen Unterschiede nicht alle im Erwerbsprozess auftretenden Normwidrigkeiten erklären. Nach ihnen seien extralinguistische Faktoren, die den Spracherwerb beeinflussen, wie Motivation, Alter oder Affektivität, bei der Kontrastivhypothese vernachlässigt (vgl. z. B. Edmondson/House 2000).
} 
stellung. Denn die Arbeit beschäftigt sich mit dem Erwerb von Verbzweitsätzen mit Subjekt im Mittelfeld bei italophonen Lernern, wobei die Bearbeitung der genannten Strukturen eine zentrale Rolle spielt. Ziel der folgenden Zusammenfassung ist es zunächst, jene kontrastiven Merkmale des Deutschen und des Italienischen in den Vordergrund zu rücken, die bei der Interpretation der in der vorliegenden Arbeit dargelegten Ergebnisse erforderlich sind, um sie dann mit den tatsächlichen Lernschwierigkeiten in Zusammenhang zu bringen.

\subsection{Verbstellung}

In der deutschen Sprache ist die Verbstellung in den einzelnen Satztypen fest. In den theoretischen bzw. didaktischen Grammatiken der deutschen Sprache wird üblicherweise der Satz nach Stellung des finiten Verbs in folgende drei Satztypen klassifiziert ${ }^{3}$.

1. Stirnsatz bzw. Verberstsatz (V[S]X):

Wirst du heute mit mir ins Kino kommen, wenn du Zeit hast?

2. Kernsatz bzw. Verbzweitsatz (SVX / XVS; [w-Part]V[S]X bei $w$-Fragen):

Ich habe das nicht vergessen.

3. Spannsatz bzw. Verbletztsatz (KonjSV):

Dass du kommst, freut mich.

Im Fall der Verbzweitsätze, auf die sich die vorliegende Arbeit beschränkt, lassen sich zwei Besonderheiten der deutschen Sprache beobachten, die im Italienischen nicht vorkommen: einerseits die Verbzweitstellung, d. h. die Stellung der konjugierten Verbform an der zweiten syntaktischen Position im Satz, andererseits die Zweiteiligkeit der deutschen Verben und ihre Fähigkeit und Tendenz, in den Sätzen Klammerstrukturen (Verbalklammern) zu bilden, wobei die linke Klammer aus dem Vorverb (dem Finitum) besteht und die rechte Klammer aus dem Nachverb (dem Verbzusatz: Präposition, Adverb, Infinitum), das die letzte Position im Hauptsatz besetzt ${ }^{4}$. Die Struktur der Verbalklammer ist in der deutschen Sprache von besonderer Bedeutung, weil sie ermöglicht, den Satz nicht nur in syntaktische, sondern auch in semantische Einheiten zu unterteilen, welche zur Wiedergabe des informativen Profils (Thema-Rhema) des Satzes (sowie des ganzen Textes, wenn dieser das Objekt der Analyse ist) dient. Durch das Vorverb (VV) und das Nachverb (NV) wird der deutsche Satz in drei topologische Felder unterteilt: das Vorfeld (VF), das Mittelfeld (MF) und das Nachfeld (NF), die nur eine partielle Entsprechung im Italienischen finden ${ }^{5}$. Das jeweilige Satzfeld kann eine variable Anzahl von Satzgliedern enthalten: Mit welchen sprachlichen Mitteln und auf welche

3 In den grammatischen Beschreibungen des Deutschen erfolgt üblicherweise die Klassifikation von Sätzen nach drei Kriterien: nach dem formalen, wobei die Satzformen einfacher Satz und zusammengesetzter Satz unterschieden werden; nach dem syntaktisch-funktionalen, bei dem Sätze nach Verbstellung in die Satztypen Stirnsatz, Kernsatz, Spannsatz unterteilt werden, und nach dem semantischen, mit welchem sich Sätze hinsichtlich verschiedener Satzarten (Aussagesatz, Fragesatz, Aufforderungssatz, Wunschsatz, Ausrufesatz) klassifizieren lassen (vgl. dazu Eisenberg 1999, Duden 2005, Dürscheid 2007).

${ }^{4}$ Im Gegensatz zu Satz tritt in den relativ neueren Beschreibungen der deutschen Sprache der Begriff Verbalklammer, die in der Duden-Grammatik (2005) Satzklammer genannt wird. Die in der vorliegenden Arbeit verwendete Terminologie bezieht sich sowohl auf die traditionelle Grammatik als auch auf die Textgrammatik und Feldertheorie (Drach 1937, Weinrich 1993), und zwar auf die traditionelle Grammatik mit den Begriffen Verbzweitstellung, Verbzweitsatz, Inversion und auf die Textgrammatik mit den Begriffen Verbalklammer, Satzfelder, Felderbesetzung.

5 Die Annahme, dass das konjugierte Verb im deutschen Satz ermöglicht, die Satzstruktur in topologische Felder unterzuteilen, hat in der Grammatikschreibung der deutschen Sprache eine relativ lange Tradition, die auf die 30er Jahre des zwanzigsten Jahrhunderts zurückgreift (Drach 1937). Drach erkennt, "dass im AussageHauptsatz überall das Geschehen in Mittelstellung gesetzt wird" und dass "das Verbum Finitum der standfeste Angelpunkt [ist], um den herum der Satz sich aufbaut und gliedert" (ebd.: 16). In der Drachschen Auffassung wird der deutsche Satz durch das konjugierte Verb, das das "Geschehen" bezeichnet, in zwei Felder aufgeteilt, nämlich Vorfeld und Nachfeld (ebd.: 17). 
Art die einzelnen Felder besetzt werden können, wird durch die Regeln der Felderbesetzung und der Satzgliedstellung bestimmt.

In der italienischen Sprache lassen sich im Gegensatz zum Deutschen bei der Verbstellung folgende Besonderheiten beobachten: 1. Abgesehen vom Satztyp (frase principale/Hauptsatz oder frase secondaria/Nebensatz) ${ }^{6}$, weist das konjugierte Verb die Tendenz auf, soweit wie möglich an den Satzanfang zu rücken (Bsp.: Andiamo a casa). Seine Stellung im Satz ist aber nicht fest: Die präverbale Position kann durch ein oder mehrere Satzglieder besetzt werden (Bsp.: Luca va a casa; Ora andiamo a casa; Oggi i ragazzi hanno lavorato seriamente); 2. das konjugierte Verb und das Subjekt (wenn dieses explizit ist) neigen dazu, einen einzigen Kern, ein Kontinuum im Satz zu bilden (S-Fin. Bsp.: Carlo mangia una mela); 3. obwohl die italienische Sprache zweiteilige Verbformen besitzt (z. B. passato prossimo), bilden diese im Satz keine Verbalklammer, weil sie fast immer aufeinander folgen (Fin.-Inf.: Siamo stati molte volte in questo ristorante; Vorrei mangiare una bella torta al cioccolato), abgesehen von einigen wenigen Ausnahmen, bei denen zwischen konjugiertem Verb und Infinitiv ein Adverb tritt (Bsp.: Non l'ho mai vista).

\section{$1.2 \quad$ Subjekt}

In der deutschen Sprache wird das Subjekt im Satz tendenziell immer expliziert (außer bei Infinitivsätzen, bei Sätzen mit Imperativformen und beim zweiten Teilsatz eines zusammengezogenen Satzes). Ein Sonderfall ist die Verwendung von es, in Sätzen wie Es wurde bis tief in die Nacht hinein diskutiert 7 : "Dieses es fungiert in unpersönlichen Passivkonstruktionen als grammatisches Subjekt. Es hat die Aufgabe, den folgenden Sachverhalt hervorzuheben und fällt weg, wenn ein anderes Element die Vorfeldposition besetzt oder wenn der Satz in eine V/E- oder einen V/1-Satz transformiert wird, also gar kein Vorfeld mehr vorhanden ist" (Dürscheid 2007: 98). Dieses Phänomen weist auf einen weiteren wichtigen Unterschied des Deutschen zum Italienischen hin: Dass es hier wohl eher eine positionssyntaktische Funktion als eine relationssyntaktische ausübt, bestätigt die Tendenz der deutschen Sprache dazu, die Realisierung von Verbzweitsätzen zu gewährleisten.

In der italienischen Sprache wird das Subjekt - wenn es nicht durch eine substantivische Nominalphrase ausgedrückt wird - tendenziell impliziert, wobei die Person und der Numerus durch das Verbalsuffix ausgedrückt werden, z. B. Dove abiti? Oggi non vengo. Desidero parlarti al più presto. Ist das Subjekt explizit, dann kommt es vorwiegend in präverbalen Position vor und neigt dazu, einen einzigen Kern, ein Kontinuum, mit dem konjugierten Verb zu bilden (Bsp.: Carlo mangia una mela). Inversionsphänomene sind selten und vom Informationsprofil abhängig (Bsp.: A condurre il censimento sono stati gli scienziati del Census of Marine Life).

\subsection{Satzgliedstellung}

In der deutschen Sprache betrifft die Satzgliedstellung die Regeln der Felderbesetzung. Typische Phänomene für die deutsche Felderbesetzung sind folgende.

\footnotetext{
6 Vom gemeinsamen lateinischen Ursprung hinaus haben die italienische und die deutsche Grammatikschreibung unterschiedliche Traditionen entwickelt und verwenden deshalb auch jeweils eine andere Terminologie. In den italienischen Grammatiken wird zwischen frase bzw. preposizione, enunciato und periodo unterschieden, im Deutschen ist der Grundbegriff der syntaktischen Definitionen durch das Wort Satz ausgedrückt, bei dem die verschiedenen Bedeutungen und semantischen Nuancen durch prä- bzw. post-determinierende Attribute bestimmt werden: einfacher Satz, komplexer Satz, Teilsatz, Satzgefüge, Satzreihe, Hauptsatz, Nebensatz, Fragesatz, Ausrufsatz (vgl. dazu auch Gallmann/Sitta in Druck).

7 Das Beispiel stammt aus Dürscheid 2007: 98.
} 
- Die Besetzung der präverbalen Position unterliegt der Regel des Vorfeldes, der zufolge die präverbale Position (das Vorfeld) in den Verbzweitsätzen nur durch ein Satzglied besetzt werden kann. Wenn in der präverbalen Position ein anderes Satzglied als das Subjekt steht, rückt das Subjekt ins Mittelfeld (Inversion) (Bsp.: Rom ist eine wunderschöne Stadt; Eine wunderschöne Stadt ist Rom $)^{8}$.

- Der Besetzung des Nachfeldes wird "eine kommunikativ-pragmatische Funktion zugeschrieben derart, dass ins Nachfeld besonders umfangreiche, semantisch gewichtige oder rhematische Satzglieder rücken" (Eisenberg 1999: 391). Im Nachfeld stehen am häufigsten Nebensätze (Adjunktklammern), Zitate und Auflistungen: Das Wetterbericht hat gemeldet, dass schönes Wetter zu erwarten ist; "Der Staat" hat der Bundespräsident Gustav Heinemann einmal gesagt, "ist nicht meine Frau"; Wir wollen zufrieden sein, heute, morgen und bis ans Ende unserer Tage (Weinrich 1993: 84).

- Komplizierter erscheint hingegen - insbesondere aus der kontrastiven Perspektive des Italienischen - zu erklären, welche Faktoren die Satzgliedabfolge im Mittelfeld bestimmen. Im Mittelfeld können nämlich mehrere Satzglieder vorkommen, es gibt keine Restriktion "außer der einen [...], dass die Klammer eine größere Dehnung verträgt, als sie vom Kontextgedächtnis verarbeitet werden kann" (Wenrich 1993: 80). Die Satzgliedstellung wird dabei nicht durch ein einziges Kriterium bestimmt, sondern es sind mehrere konkurrierende Regularitäten feststellbar, wie beispielsweise die Anordnung nach syntaktischer Funktion (Subjekt vor dem Objekt), nach Thema-Rhema-Struktur (Thema vor Rhema), nach Satzakzent (akzentuiertes nach unakzentuiertem Satzglied), nach Form des Satzgliedes (pronominale vor substantivischer Nominalphrase), nach Bedeutung des Satzgliedes (definites vor undefinitem Satzglied; belebtes vor unbelebtem Satzglied, Start vor Ziel), nach Länge der Satzglieder (kurzes vor langem Satzglied) (vgl. dazu Weinrich 1993, Zifonun et al. 1997, Eisenberg 1999, Dudenredaktion 2005). Im Allgemeinen wird die Satzgliedstellung gleichzeitig durch formale (grammatische) und pragmatisch-semantische Regeln gesteuert. Ein wichtiger Unterschied zwischen dem Italienischen und dem Deutschen sind die Determinationsprinzipien (z. B. die Stellung der Negationsmorpheme und der Modalpartikeln) ${ }^{9}$.

Da es im italienischen Satz keine Verbalklammer gibt, können dabei keine Satzfelder unterschieden werden, sondern eine präverbale und eine postverbale Position. Die Satzgliedabfolge im Italienischen weist sowohl Unterschiede als auch Ähnlichkeiten zum Deutschen auf. Anders als im Deutschen kann die präverbale Position leer sein (Bsp.: Parto alle tre), ein Satzglied (Bsp.: Questa volta parto alle tre; Carlo parte alle tre) oder mehrere Satzglieder (Bsp.: Questa volta I Carlo parte alle tre) enthalten. Die Satzgliedabfolge in der postverbalen Position, die dem Mittelfeld und dem Nachfeld des deutschen Satzes entspricht, wird durch Regeln gesteuert, die denjenigen des deutschen Mittelfeldes ähnlich sind. Im Italienischen wie im Deutschen wird die Satzgliedstellung durch die Miteinwirkung von formalen und pragmatisch-semantischen Regeln bestimmt. Die Tatsache, dass zum einen Mittelfeld und Nachfeld einem einzigen "Satzfeld" (der postverbalen Position) entsprechen, und zum anderen dass das Deutsche und das Italienische verschiedene Determinationsprinzipien aufweisen, führt allerdings praktisch zur Realisierung zweier unterschiedlicher Satzstrukturen.

Den bisher vorgenommenen Untersuchungen zufolge entsprechen die oben beschriebenen Unterschiede den Fällen, bei denen italophone Lerner besondere Erwerbsschwierigkeiten

8 Es handelt sich um die häufigste Tendenz, es gibt aber auch Ausnahmen. In einigen Fällen kann z. B. das Vorfeld des Deklarativsatzes doppelt besetzt sein, wie im Fall des Vor-Vorfeldes im Satz Zum Beispiel: Rom ist eine wunderschöne Stadt (vgl. z. B. Eisenberg 1999: 388; Dürscheid 2000: 97-101).

9 Dabei folgt dem Informationsprofil auch in den meisten Fällen das Intonationsprofil (vgl. z. B. dazu Weinrich 1993: 81) 
aufweisen. Deswegen werden im vorliegenden Beitrag Verbzweitsätze mit Subjekt im Mittelfeld unter die Lupe genommen. Es handelt sich dabei um einen grammatischen Bereich, der von mehreren Autoren untersucht wurde und bei dem sich sowohl Gemeinsamkeiten als auch Unterschiede zwischen den jeweiligen Lernergruppen feststellen lassen.

Laut diesen Resultaten stellt bei italophonen Lernern die Verinnerlichung der Struktur "Verbzweitsatz mit Subjekt im Mittelfeld" einen wichtigen Schwerpunkt im Grammatikerwerb des Deutschen dar: Während der Erwerb der VS-Ordnung bei anderen Satzmodellen ( $w$ - und Entscheidungsfragen) schon in den ersten Stadien des Erwerbsprozesses erfolgt, erweist sich der Erwerb der XVS-Ordnung bei Verbzweitsätzen als vergleichsweise schwierig. Grund dafür können folgende zwei Faktoren sein:

1. Die normkonforme Verwendung eines solchen Satzmodells setzt die Beherrschung einer spezifisch deutschen Regel voraus, die im Italienischen nicht existiert, d. h. der Regel der Verbzweitstellung in Verbzweitsätzen.

2. Hinzu kommt, dass der Erwerb dieser Regel mit dem Erwerb der Distanzstellung in den Verbalklammern zeitlich zusammenfällt. Sowohl die Verbzweitstellung als auch die Distanzstellung sind für italophone Lerner aus der L1 unbekannte Strukturen. Die Interferenz der Muttersprache würde zudem auch zwei Unterschiede zwischen dem L1- bzw. L2-Erwerb des Deutschen erklären.

Der L2-Erwerb von Verbzweitsätzen mit XVS-Ordnung erfolgt bei italophonen Lernern sowohl bei erwachsenen Gastarbeitern (Clahsen et al. 1983) als auch bei Schulkindern und Jugendlichen (Terrasi Haufe 2000) - erst in den letzten Phasen des Erwerbsprozesses, obwohl diese Satzform von Anfang an im Input vorhanden ist. Dieses Ergebnis spricht gegen die Identitätshypothese und für die kontrastive, da im L1-Erwerb (Clahsen 1982) die Verbzweitstellung schon in den ersten Erwerbsphasen beherrscht wird.

Dass die Muttersprache den Erwerb der Syntax beeinflusst, wird außerdem durch das Forschungsergebnis bestätigt, dem zufolge der Transfer aus der L1 die Lernstrategie ist, die von den italophonen Lernern bei der Bearbeitung der deutschen syntaktischen Strukturen am häufigsten eingesetzt wird, und zwar in allen Phasen des Erwerbsprozesses. Das betrifft sowohl den natürlichen (Clahsen et al. 1983) als auch den gesteuerten Erwerb (Terrasi Haufe 2004).

In der vorliegenden Arbeit geht es nicht darum, die durch die vorhergehenden Studien unterstützte kontrastive These zu widerlegen, sondern es soll mit weiteren Beobachtungen zur Bestätigung ihrer Gültigkeit sowie zur Reflexion über bestimmte Besonderheiten des Spracherwerbsprozesses beigetragen werden. Anhand der Resultate einer empirischen Untersuchung zum DaF-Erwerb ausgewählter grammatischer Strukturen des Deutschen bei Studierenden der Universität Pisa (Ballestracci 2006) kann folgendermaßen argumentiert werden:

1. die muttersprachlichen Vorkenntnisse spielen im Erwerb von syntaktischen Zielstrukturen die wichtigste Rolle (Kap. 2) ${ }^{10}$;

\footnotetext{
${ }^{10}$ Bei der Pisaner Untersuchung wurden auch andere grammatische Bereiche untersucht: die Verbalmorphologie und die Kasus-Deklination der Nominalphrasen. Die Entscheidung, bestimmte grammatische Bereiche zu fokussieren, orientiert sich an dem Projekt Deutsch in Genfer Schulen von Diehl et al. (2000). Dabei haben sich die Autorinnen auch mit der Genuszuweisung, der Pluralbildung und den Präpositionalphrasen beschäftigt. Eine Phasenabfolge wurde aber nur im Erwerbsprozess der Verbalmorphologie, der Kasus-Deklination in Nominalphrasen und der syntaktischen Strukturen beobachtet. Die Pisaner Untersuchung überprüfte, ob die Phasen des DiGS-Projektes auch für italophone Studierende gelten. Die Analyse ergab, dass es auch bei den italophonen Lernern eine Phasenabfolge gibt, jedoch mit Unterschieden in Bezug auf die Dauer der Phasen und der Reihenfolge der zu erwerbenden Strukturen. Diese Unterschiede zwischen den Erwerbsphasen der frankophonen Schulkinder und jenen der Pisaner Studierenden können durch das Auftreten bestimmter Variablen (Muttersprache, sozialer Kontext, Bildung, Motivation, didaktischer Input) erklärt werden.
} 
2. die Lerner setzen auch andere Sprachverarbeitungsstrategien beim Erwerb dieser Struktur ein, nämlich Chunks und Verwechslungen. Durch die Analyse der Art und Weise, wie solche Strategien benutzt werden, wird auf die Frage eingegangen, ob sich ihr Auftreten ebenfalls durch den Einfluss der Muttersprache erklären lässt oder ob hier die Mitwirkung anderer Einflussfaktoren anzunehmen ist. Dabei scheinen die Variablen "Gedächtnis", "Sprachbewusstheit" und "didaktischer Input" eine wichtige Rolle im Spracherwerbsprozess zu spielen (Kap. 2);

3. aus den gewonnenen Ergebnissen können wertvolle Schlussfolgerungen für die didaktische Praxis des Deutschen als L2 gewonnen werden (Kap. 3). Am Beispiel des Erwerbs der Grundstrukturen des deutschen Satzes wird gezeigt, wie die Interferenzen aus der L1, das Chunk-Learning und die Strategie der Verwechslungen als positive Einflussfaktoren beim gesteuerten Erwerbsprozess angewandt werden können. In der Forschung ist es unumstritten, dass die hier genannten Variablen am Spracherwerbsprozess beteiligt sind: Was noch fehlt - zumindest in Italien - ist der Versuch, die durch empirische Untersuchungen gewonnenen Ergebnisse in der didaktischen Praxis in adäquate Lehrmethoden bzw. -techniken umzusetzen.

\section{Forschungsergebnisse zum Erwerb von Verbzweitsätzen mit Subjekt im Mittelfeld bei italophonen Lernern}

Wie schon erwähnt, geht aus den bisher verfügbaren Studien zum L2-Erwerb des Deutschen bei italophonen Lernern hervor, dass der Erwerb von Verbzweitsätzen mit XVS-Ordnung durch die Interferenzen aus der Muttersprache stark beeinflusst ist. Beim Vergleich der bei den jeweiligen Untersuchungen gewonnenen Ergebnisse zeigen sich aber auch einige Unterschiede, die vermuten lassen, dass auch andere, extra-linguistische Faktoren dabei mitwirken. Um zu überprüfen, ob und - wenn ja welche - andere/n Variable/n den Erwerb dieser Struktur beeinflussen, werden in der Folge die Resultate dreier Untersuchungen zum Erwerb des Deutschen als L2 bei italophonen Muttersprachlern besprochen:

1. das Projekt Zweitsprachenerwerb italienischer und spanischer Arbeiter (ZISA-Projekt) von Clahsen, Meisel und Pienemann (1983), das den Erwerb der Wortstellung im Deutschen bei 45 italienisch-, spanisch- und portugiesisch-sprachigen Gastarbeitern untersucht; ${ }^{11}$

2. die Studie Der Schulerwerb von Deutsch als Fremdsprache. Eine empirische Untersuchung am Beispiel der italienischsprachigen Schweiz von Haufe Terrasi (2004);

3. die empirische Untersuchung Zum DaF-Erwerb ausgewählter grammatischer Strukturen der deutschen Sprache bei italophonen Studierenden der Pisaner "Facoltà di Lingue e Letterature Straniere" (Ballestracci 2006).

\subsection{Verbstellung}

Die Autoren des ZISA-Projektes haben die ersten wichtigen Ergebnisse zu diesem Gegenstand beschrieben, indem sie durch eine empirische Untersuchung bei romanischsprachigen Lernern feststellten, dass das Lernen der deutschen syntaktischen Strukturen über sechs implikationell angeordnete Phasen verläuft, d. h.:

\footnotetext{
${ }^{11}$ Die Erhebung der Daten erfolgte hier durch ungeleitete Interviews, die mit einem Audiorecorder aufgenommen und dann transkribiert wurden. Für jeden Lerner wurden 50 Äußerungen ausgewählt (vgl. dazu auch Clahsen 1980).
} 
1. SVO: das Verb folgt dem Subjekt und geht dem Objekt voran

2. ADV-VOR: dem Subjekt wird fakultativ ein Satzglied (Adverbial) vorangestellt, das Verb rückt an die dritte Stelle

3. PARTIKEL: der nicht finite Teil der Verbform steht am Satzende

4. INVERSION: das Subjekt folgt der finiten Verbform in Fragesätzen, nach vorangestellten Adverbialen, Objekt-Nominalphrasen und Nebensätzen

5. ADV-P: fakultative Stellung von Adverbialen zwischen Verb und Objekt

6. V-ENDE: finite Verbform steht im Nebensatz am Satzende

Außerdem hat das Wuppertaler Forschungsteam beobachtet, dass die Lerner in jeder Phase besondere Sprachverarbeitungsstrategien einsetzen, d. h.:

1. die Canonical Order Strategy (COS), die keine Permutationen von semantisch zusammengehörigen Elementen zulässt und auf der die Wortfolge der Phase I (SVO) und II (ADV-Preposing) basiert,

2. die Initialization/Finalization Strategy (IFS), der zufolge Permutationen am Satzanfang und Satzende möglich sind (Phase III),

3. die Subordinate Clause Strategy (SCS), die Permutationen in Nebensätzen blockiert, nicht aber in Hauptsätzen (Phase IV: die Inversion) (vgl. dazu Clahsen et al. 1983: 158).

Die hierbei gewonnenen Ergebnisse weisen darauf hin, dass der Erwerb von Verbzweitsätzen mit Subjekt im Mittelfeld den romanischsprachigen Lernern besondere Schwierigkeiten bereitet und dass ihre Verwendung durch die L1 beeinflusst ist. Die erste Tatsache bestätigt sich dadurch, dass die Bearbeitung dieser Satzform nicht in einer einzigen Phase, sondern in mehreren (von der zweiten bis zur vierten Phase) erfolgt. Der Einfluss der L1 wird dadurch deutlich, dass die am Anfang des Erwerbsprozesses verwendeten Satzstrukturen die gleiche Satzgliedstellung wie im Italienischen aufweisen. Dies führt zur Verwendung von normkonformen Sätzen, wenn die deutsche Struktur mit jener der Muttersprache übereinstimmt, z. B. im Fall von Verbzweitsätzen mit SVX-Ordnung:

$[\mathrm{Z} 1]^{12}$ die kinder spielen mim ball

und zur Verwendung von normwidrigen Sätzen, wenn die deutsche Struktur nicht mit jener der L1 identisch ist, z. B. im Fall von Verbzweitsätzen mit vorangestelltem Adverbial, in denen das Verb an die dritte Stelle rückt:

[Z 2] hier in den nächsten tagen kaufe ich das auto

Die normkonforme Verwendung von Verbzweitsätzen mit vorangestelltem Adverbial erfolgt erst in der vierten Phase, d. h. nachdem die Distanzstellung der Verbalklammer erworben ist. Bsp.:

[Z 3] jetzt gehe ich nach hause

[Z 4] ich habe funf klass gemacht

\subsection{Subjekt}

$\mathrm{Zu}$ ähnlichen Ergebnissen kommt auch Terrasi Haufe in ihrer Untersuchung zum Erwerb des Deutschen als L2 durch italophone Lerner an einer Tessiner Schule. Hier wird die Schwierig-

12 Die Sigle Z heißt ZISA, aus dem gleichnamigen Projekt von Clahsen, Meisel und Pienemann, aus deren Korpus die Beispielsätze stammen. 
keit italophoner Lerner bestätigt, Verbzweitsätze mit XVS-Ordnung zu erwerben, und wird mit dem Einfluss der L1 erklärt (vgl. Terrasi Haufe 2004: 113-128).

Bei den Tessiner Probanden ergeben sich allerdings kleine, aber wichtige Unterschiede zu den Ergebnissen des ZISA-Projektes. Zunächst soll erwähnt werden, dass Terrasi Haufe mehr Inversionskontexte als Clahsen, Meisel und Pienemann unterscheidet, d.h. Inversion in Ergänzungsfragen, in Entscheidungsfragen, nach vorangestelltem Nebensatz und nach vorangestelltem Adverbial bzw. einleitendem Konjunktionaladverb. So konnte die Autorin genauer beobachten, bei welchen Satzstrukturen die schwersten Lernblockaden vorkommen. Terrasi Haufe stellt folgende Erwerbsphasenabfolge fest:

1. Subjekt-Verb-Objekt

2. Inversion in Ergänzungsfragen

3. Negation

4. Distanzstellung

5. Inversion in Entscheidungsfragen

6. Inversion nach vorangestellten Nebensätzen

7. Verbendstellung in infiniten Nebensätzen

8. Verbendstellung in finiten Nebensätzen

9. Inversion nach topikalisierten Adverbialen

10.Inversion nach einleitenden Konjunktionaladverbien

Die gewonnenen Ergebnisse bestätigen, dass Lernschwierigkeiten in Sätzen mit Inversion nach topikalisiertem Adverbial bzw. einleitendem Konjunktionaladverb vorkommen, während die Inversion in anderen Kontexten (Ergänzungs- bzw. Entscheidungsfragen) offenbar schneller beherrscht wird (vgl. Terrasi Haufe 2004: 116):

[TH 5 $]^{13}$ Am Anfang dieser Projekt wird patentiert.

[TH 6] wenn die Wissenschaftler nicht mehr mit ihren Arbeiten weitergehen, das wird unmöglich, die Krankheiten zu schlagen.

[TH 7] Mir die Natur schmeckt so [...]

Außerdem zeigt sich bei Terrasi Haufe ein weiterer Unterschied zu den ZISA-Ergebnissen: Im Gegensatz zu den eingewanderten Gastarbeitern erwerben die italophonen Schüler zuerst die Struktur des Nebensatzes und danach jene der Inversion. Ein Ergebnis, das mit jenem eines anderen Forschungsprojekts übereinstimmt: Das Projekt Deutsch in Genfer Schulen (Diehl et al. 2000), bei dem der L2-Erwerb frankophoner Schulkinder der Genfer Schulen untersucht wurde. Die Unterschiede zwischen den ZISA-Ergebnissen einerseits und den Tessiner bzw. Genfer Ergebnissen andererseits können durch den Einfluss folgender Aspekte erklärt werden:

- Erwerbstyp. Beim ZISA-Projekt handelt es sich um natürlichen Erwerb, bei Diehl et al. und bei Terrasi Haufe um gesteuerten Erwerb, obwohl die Lerner der Genfer und Tessiner Schulen in einer mehrsprachigen Situation leben, weshalb Kontakte mit dem deutschsprachigen Raum möglich sind.

- Alter. Die Testpersonen des ZISA-Projekts sind Erwachsene, die frankophonen und italophonen Schüler Kinder und Jugendliche.

${ }^{13}$ Die Sigle TH heißt Terrasi Haufe. Auch in diesem Fall stammen die Beispielsätze aus dem originalen Werk der Autorin. 
- Mündlichkeit vs. Schriftlichkeit. Die Korpora der drei Projekte sind unter diesem Aspekt nur teilweise vergleichbar. Im Gegensatz zum ZISA-Korpus, das nur mündliche Texte enthält, besteht das DIGS-Korpus ausschließlich aus schriftlichen Arbeiten. Bei Terrasi Haufe handelt es sich um ein zweiteiliges Korpus, wobei ein Teil aus mündlichen und ein Teil aus schriftlichen Texten besteht.

\subsection{Satzgliedstellung}

$\mathrm{Zu}$ ähnlichen, aber nicht identischen Ergebnissen kommt auch die empirische Untersuchung, die bei italophonen DaF-Studierenden an der Universität Pisa auf der Basis eines Korpus schriftlicher Arbeiten vorgenommen wurde.

Das Pisaner Korpus besteht aus 157 durch Null-Anfänger ${ }^{14}$ verfassten Arbeiten (Typ: freies Schreiben), das sich in drei Sektionen (Korpus I, II und III) und acht chronologische Phasen (Zeitpunkte der Datenerhebung) unterteilt. Jede Sektion enthält die schriftlichen Arbeiten einer bestimmten Student/Innen-Gruppe: Korpus I besteht aus den schriftlichen Arbeiten der Studierenden des ersten Jahres 2001/2002, Korpus II aus den schriftlichen Arbeiten der Studierenden des ersten Jahres 2002/2003 und Korpus III aus den schriftlichen Arbeiten der Studierenden des zweiten Jahres 2001 bzw. 2004. Die Datenerhebung erfolgte: viermal im ersten Jahr (nach 40, 80, 120 und 160 Unterrichtsstunden), dreimal im zweiten Jahr (nach 200, 240 und 320 Unterrichtsstunden) und einmal am Ende des dritten Jahres (nach 440 Unterrichtsstunden).

Durch die Analyse der vorkommenden Strukturen und des Verhältnisses zwischen ihrem normkonformen bzw. normwidrigen Auftreten wurden innerhalb des jeweils untersuchten Bereiches bestimmte Erwerbsphasen ${ }^{15}$ unterschieden. Mit "Erwerbsphasen" sind hier die Ausschnitte gemeint, in die der Erwerbsprozess der italophonen Studierenden in Pisa unterteilt werden kann. Die Unterteilung des Erwerbsprozesses in Erwerbsphasen erfolgt dabei durch bestimmte Kriterien.

$\mathrm{Zu}$ bestimmen, wann eine Struktur als erworben gilt, ist allerdings problematisch; es kommt nämlich oft vor, dass eine Struktur, die zu einem gewissen Zeitpunkt fast immer normkonform, dann später wieder normwidrig verwendet wird. Deswegen verstehe ich unter Erwerbsphasen die sequentiellen Ausschnitte, in denen die Bearbeitung einer oder mehrerer grammatischer Strukturen innerhalb der einzelnen grammatischen Bereiche erfolgt ${ }^{16}$. In den einzelnen untersuchten Bereichen können die folgenden Erwerbsphasen unterschieden werden: vier Erwerbsphasen bei der Verbalmorphologie, fünf Erwerbsphasen bei der Satzgliedstellung und fünf Erwerbsphasen bei der Kasus-Deklination der Nominalphrasen ${ }^{17}$. Wie in Tab. 1 veranschaulicht wird, gehört die Inversion bei der Satzgliedstellung in die vierte Erwerbsphase.

\footnotetext{
${ }^{14}$ Um die "echten" Null-Anfänger zu erkennen, wurde unter den Studierenden ein Fragebogen verteilt, in dem die Probanden unter anderem auch um eine Selbsteinschätzung ihrer Sprachkenntnisse ersucht wurden. Dank der 419 analysierten Fragebögen war es möglich, die Null-Anfänger unter den Verfassern der insgesamt 300 gesammelten Arbeiten herauszufinden.

15 Hier soll betont werden, dass die Erwerbsphasen nicht mit den "chronologischen Phasen" übereinstimmen, welche die acht Zeitpunkte sind, zu denen die Bestandsaufnahme stattfand (zur Definition von Erwerbsphasen in der Pisaner Untersuchung vgl. auch Ballestracci 2008: 164-167).

16 Jede Phase lässt sich dann weiter in Stadien unterteilen: Die Stadien entsprechen kleineren sequentiellen Einheiten, in denen ein bestimmter Aspekt der zu erwerbenden Struktur bearbeitet bzw. eine bestimmte Lernstrategie angewandt wird.

${ }^{17}$ In allen untersuchten grammatischen Bereichen wurde eine letzte Erwerbsphase im Erwerbsprozess angenommen, nämlich eine Phase der Konsolidierung. In dieser Phase sollte sich der Erwerbsprozess so vollziehen, dass zunächst der Ausbau und dann die Konsolidierung der gelernten Strukturen erfolgt, da nicht alle Lerner am Ende des Beobachtungszeitraums imstande sind, alle vermittelten Strukturen normkonform zu verwenden.
} 


\begin{tabular}{|c|c|c|}
\hline Phasen & Erwerbsphasen bei der Satzgliedstellung & $\begin{array}{l}\text { Erwerbsstadien der Verbzweitsätze } \\
\text { mit Subjekt im Mittelfeld }\end{array}$ \\
\hline I & $\begin{array}{l}\text { Deklarative Hauptsätze mit S-V-Struktur } \\
w \text {-Frage }\end{array}$ & \multirow{2}{*}{$\begin{array}{l}\text { Chunks } \\
\text { pro-drop-Phänomen } \\
\text { Interferenzen aus der L1 }\end{array}$} \\
\hline II & E-Frage & \\
\hline III & Distanzstellung (Verbalkomplex) & Interferenzen aus der L1 \\
\hline IV & \multirow{4}{*}{$\begin{array}{l}\text { Nebensatz } \\
\text { Inversion }\end{array}$} & \multirow{4}{*}{$\begin{array}{l}\text { Interferenzen aus der L1 } \\
\text { Verwechslung }\end{array}$} \\
\hline $\mathrm{V}$ & & \\
\hline $\mathrm{VI}$ & & \\
\hline VII & & \\
\hline VIII & Konsolidierung & normkonforme Verwendungen \\
\hline
\end{tabular}

Tab. 1: Verhältnis zwischen chronologischen Phasen, Erwerbsphasen der Satzgliedstellung und Erwerbsstadien der Verbzweitsätze mit Subjekt im Mittelfeld ${ }^{18}$.

Die beim Pisaner Projekt gewonnenen Ergebnisse bestätigen großteils die Resultate von Terrasi Haufe (und analog jene von Diehl et al. 2000) und des ZISA-Projekts. Die Bearbeitung der Deklarativsätze ${ }^{19}$ verläuft über vier Erwerbsstadien und erstreckt sich über den ganzen Erwerbsprozess. Das erste Erwerbsstadium entspricht den chronologischen Phasen III, das zweite der chronologischen Phase III, das dritte den chronologischen Phasen IV-VII und das vierte Stadium der letzten chronologischen Phase (Konsolidierung). Auch bei den Pisaner Studierenden wird wie bei den Tessiner Lernern und den Wuppertaler Gastarbeitern zuerst die Satzstruktur mit SVX-Ordnung bearbeitet. In der ersten chronologischen Phase werden 768 Deklarativsätze (81,88\% aller vorkommenden Sätze) verwendet. 94,4\% weisen SVX-Struktur auf.

[B 8] Das Foto ist charmant und wunderbar.

Ich sehe zwei Personen. Sie sind ein Alt und eine Kleine. (I.cP/48) ${ }^{20}$

Nur 5,6\% aller Deklarativsätze haben XVS-Struktur und nur 1,8\% davon sind normkonform.

[B 9] Die habe ich schon gewäscht. (I.cP/101)

Dass die XVS-Struktur in einigen Fällen normkonform verwendet wird, kann aber noch nicht bestätigen, dass die Regel der Verbzweitstellung schon erworben ist. Als Beweis dafür gilt die Tatsache, dass der Lernende, der in [B 9] die Regel der Verbzweitstellung normkonform benutzt, dieselbe Regel an anderen Stellen im gleichen Text nicht beachtet.

[B 10] Im das Photo ich sehe ein Großvater und ein Kind. (I.cP/101)

Dieses Phänomen, das auch in der zweiten chronologischen Phase auftritt, lässt vermuten, dass die Regel der Verbzweitstellung noch nicht verinnerlicht ist. Es handelt sich dabei mit einer gewissen Sicherheit um eine Struktur, die während des Unterrichts im Gedächtnis gespeichert und danach reproduziert wurde, wobei der Lerner jedoch nicht imstande ist, die hinter der Wortfolge verborgene Regel zu begreifen. Dieses Ergebnis kann als eine Bestäti-

\footnotetext{
${ }^{18}$ Dabei wird verdeutlicht, dass sich der Erwerb der Verbzweitsätze über den ganzen Erwerbsprozess erstreckt und in vier Stadien unterteilt ist: In den einzelnen Stadien kommen verschiedene Lernschwierigkeiten bzw. strategien vor.

${ }^{19}$ In der Pisaner Untersuchung wird unter Satz ein Teilsatz verstanden, nämlich jede Satzeinheit, die eine konjugierte Verbform enthält. Zur Gruppe der Deklarativsätze gehören also einfache Hauptsätze, Teilsätze in Satzreihen bzw. in zusammengezogenen Sätzen und die Hauptsätze in zusammengesetzten Sätzen.

${ }^{20}$ I.cP/48: I.cP heißt erste chronologische Phase, 48 ist die Nummer, mit der der Studierende identifiziert wird.
} 
gung dafür gelten, dass das Chunking beim Erwerb von unbekannten L2-Strukturen eine wichtige Rolle spielt.

In der dritten chronologischen Phase werden 270 Deklarativsätze (72,78\% aller vorkommenden Sätze) verwendet. Hier fangen die Studierenden an, die Regel der Verbzweitstellung bewusst zu bearbeiten, da diese Regel induktiv im Unterricht eingeführt und im Grammatikunterricht und in den theoretischen Kursen geübt wurde. 20,74\% der Sätze haben XVSOrdnung; 19,63\% davon sind normkonform.

[B 11] Zum Gluck es war mein Geburtstag (IIIcP/99)

[B 12] Als ich die Tür meinen Hauses geöffnen habe, ich habe gerutschet (IIIcP/100)

Von der vierten bis zur siebten chronologischen Phase ist der Erwerb von Deklarativsätzen mit Subjekt im Mittelfeld durch andere Strategien als in den ersten Phasen gekennzeichnet: Die Lerner assoziieren die Regel der Verbzweitstellung mit jener der Verbendstellung in den Nebensätzen, so dass die zwei Strukturen miteinander verwechselt werden, d. h. es werden Deklarativsätze mit Verbendstellung und Nebensätze mit Verbzweitstellung benutzt.

[B 15] Wir verbrachten 15 Tagen dort, und denn wir nach Haus wieder fuhren (IVcP/101)

[B 16] Aus seinem Wörtern geht hervor, dass die Strickarbeiten ist die häufigste Arbeit (VIIIcP/101)

Bei den Pisaner Lernern ist es nicht möglich, wie bei den Tessiner DaF-Lernern und den ZISA-Gastarbeitern, eine eindeutige Phasenabfolge im Erwerb von Inversion/Nebensatz festzustellen, da diese beiden Strukturtypen in den letzten schriftlichen Arbeiten immer noch normwidrig verwendet und sehr oft miteinander verwechselt werden. Deklarativsätze mit Inversion werden jedoch von den Pisaner Studierenden in der letzten chronologischen Phase häufiger normkonform verwendet als Nebensätze mit Verbendstellung, was vermuten lässt, dass der Erwerb der Inversion in einer hier nicht belegten zukünftigen Phase vor dem Erwerb der Verbendstellung erfolgt. In diesem Sinne stehen die Pisaner Resultate den ZISAErgebnissen näher als den Ergebnissen von Terrasi Haufe.

Welche Faktoren das Auftreten dieser kleinen, aber wichtigen Ähnlichkeiten und Unterschiede beeinflussen, kann hier durch den Vergleich der extralinguistischen Variablen bestimmt werden, die den Erwerbsprozess bei den drei Lernergruppen charakterisieren.

- Muttersprache. Die Muttersprache ist bei allen Lernergruppen gleich, sodass sie als Grund für die gemeinsamen Lernschwierigkeiten und -strategien angesehen werden kann. Dazu gehört das Phänomen der Interferenz aus der L1.

- Erwerbstyp. Bei den Pisaner Studierenden und bei den Tessiner Schulkindern und Jugendlichen erfolgt der Erwerbsprozess unter gesteuerten Bedingungen, beim ZISA-Projekt hingegen unter natürlichen. Die Ähnlichkeit zur ersten Lernergruppe und der Unterschied zur zweiten zeigen, warum einige Phänomene (z. B. die Verwechslungen) vor allem im didaktischen Kontext auftreten. Dieses lässt sich dadurch erklären, dass der Input durch die Vermittlung grammatischer Regeln gegeben ist, d.h. durch eine bewusste Reflexion über die Sprache. Auch die Bearbeitung bzw. Verinnerlichung der Regeln durch die Lerner erfolgt auf einer bewussten Ebene: Eben die bewusste Verarbeitung wäre der Grund dafür, dass zwei verschiedene, aber irgendwie ähnliche grammatische Strukturen, wie beispielsweise die Verbzweitstellung und die Verbendstellung, im gesteuerten Spracherwerb häufiger als im natürlichen miteinander verwechselt werden ${ }^{21}$.

\footnotetext{
${ }^{21}$ Aus gleichem Grund wäre hingegen im natürlichen Erwerb das Chunk-Learning die bevorzugte Lernstrategie, durch die die L2-Strukturen, obwohl unanalysiert, trotzdem in ihrer authentischen Verwendungsweise memorisiert werden.
} 
- Alter. Diese Variable ist ein dem Pisaner und dem ZISA-Projekt gemeinsamer Faktor: Bei den Lernern handelt es sich - im Unterschied zu den Kindern und Jugendlichen der Tessiner Untersuchung - um Erwachsene. Das Alter erweist sich als eine plausible Erklärung für jene Phänomene, die bei den Pisaner Studierenden und den ZISA-Probanden, jedoch nicht bei den Tessiner Schülern auftreten. Dazu gehört die Phasenabfolge im Erwerbsprozess der Strukturen "Inversion" und "Nebensatz": Es handelt sich um zwei Grundstrukturen der deutschen Sprache, die im Italienischen keine Entsprechung haben. Der Faktor "Alter" kann ein Grund für diese Lernschwierigkeit sein, die bei den Erwachsenen manchmal auch zu Fossilisierungs- und Regressionsphasen führt. Denn das neuronale System von Erwachsenen verfügt über eine geringere Flexibilität als jenes der Kinder, was sich auf den Lernerfolg beim Erwerb neuer Strukturen auswirkt (vgl. dazu Dulay et al. 1982).

\section{$3 \quad$ Fazit und didaktische Implikationen}

Die dargelegten Ergebnisse zeigen, dass der Erwerb von Deklarativsätzen mit XVS-Ordnung bei allen italophonen Lernern durch Interferenzen aus der L1 beeinflusst ist, wobei bei den Probanden der Pisaner Studie durch die Aspekte Alter (erwachsene Lerner) und Erwerbstyp (gesteuerter Erwerb) Unterschiede hinsichtlich der Lernstrategien erklärt werden können. In Bezug auf das Lernen von Deutsch als Fremdsprache durch italienische Studierende kann man folgende Ergebnisse zusammenfassen. Zunächst werden vier Erwerbsphasen unterschieden:

1. In der ersten Erwerbsphase (die den chronologischen Phasen I-II entspricht, vom Anfang bis 80 Unterrichtsstunden) werden vor allem Sätze verwendet, die die gleiche Satzgliedstellung wie im Italienischen aufweisen (SVX). Dabei erfolgt ein sogenannter positiver Transfer, da die Struktur der L1 mit jener der L2 übereinstimmt. Sehr selten bilden die Lerner Deklarativsätze mit XVS-Ordnung, wobei es sich entweder um Chunks oder um normwidrige Verwendungen handelt.

2. In der zweiten Erwerbsphase (chronologische Phasen III-IV, von 80 bis 120 Unterrichtsstunden) beginnt in allen grammatischen Bereichen die Bearbeitung jener Strukturen der L2, die keine Entsprechung in der L1 finden: Bei der Syntax setzen sich die Lerner mit den spezifischen Satzstrukturen des Deutschen auseinander, die im Italienischen eine andere Satzgliedstellung haben. Dabei wenden sie bestimmte Lernstrategien an. Die häufigste Lernstrategie ist der negative Transfer aus der L1: Bei Deklarativsätzen mit vorangestelltem Adverbial werden Strukturen der L1 mit fehlendem Subjekt bzw. mit konjugiertem Verb an der dritten Stelle konstruiert.

3. In der dritten Erwerbsphase (chronologische Phasen V-VII, von 120 bis 320 Unterrichtsstunden) ist der Erwerb von Deklarativsätzen mit XVS-Ordnung mit jenem der Verbendstellung bei den Nebensätzen verbunden. Dabei tritt eine andere Lernstrategie auf: die Verwechslung oder auch "intralinguale Interferenz" genannt.

4. Die vierte Erwerbsphase (chronologische Phase VIII, von 320 bis 440 Unterrichtsstunden) entspricht der Phase des Ausbaus und der Konsolidierung, die - wie schon erwähnt - nicht von allen Studierenden erreicht wird.

Da der Deklarativsatz mit XVS-Ordnung ein Schwerpunkt des DaF-Erwerbs bei italophonen Studierenden und auch eine der Grundstrukturen des Deutschen ist, sollte diese Struktur von Anfang an in den Unterricht eingeführt werden. Die Frage ist, welche Lehrstrategien für den universitären DaF-Unterricht bei italophonen Null-Anfängern adäquat sind. Bei den hier empfohlenen Lehrstrategien, werden zwei Punkte berücksichtigt: einerseits die Lernschwierigkeiten und -strategien und andererseits die Modalitäten des DaF-Unterrichts an den 
italienischen Universitäten. Der erste Punkt wurde im vorliegenden Beitrag schon weitgehend beschrieben. Ziele und Modalitäten des DaF-Unterrichts in Italien können hingegen folgendermaßen zusammengefasst werden: Die italienische universitäre DaF-Didaktik zielt darauf ab, nicht nur sprachliche Fähigkeiten (Sprechen, Hören, Lesen und Schreiben) in der L2 zu entwickeln, sondern auch eine bewusste Kenntnis der L2-Grammatik und allgemeine metasprachliche Kompetenzen zu vermitteln. Zu diesem Zweck verfügen fast alle italienischen Fakultäten, an denen die deutsche Sprache unterrichtet wird, über zwei Typen von Kursen: theoretische Kurse in Lingua Tedesca und Kurse des Lektorats. ${ }^{22}$ Dabei soll aber berücksichtigt werden, dass an fast allen italienischen Universitäten die Mehrzahl der Studierenden Null-Anfänger in der deutschen Sprache ist. In den sechs zur Verfügung stehenden Semestern sollen sie das Sprachniveau C1 des Gemeinsamen Europäischen Referenzrahmens (GER) erreichen.

Aufgrund dieser Beobachtungen ergeben sich folgende wichtige didaktische Empfehlungen für den universitären DaF-Unterricht bei italophonen Studierenden ${ }^{23}$ :

- In der ersten Erwerbsphase erfolgt der erste Kontakt mit der Zielsprache. Die Vermittlung der Regeln der Verbzweitstellung und der Verbalklammer ist in dieser Phase wichtig, damit den Studierenden von Anfang an klar ist, wie die Grundstruktur des deutschen Satzes aussieht. Die vorgenommenen Untersuchungen bestätigen, dass die erste Phase durch Transfer aus der L1 und Chunk-Learning charakterisiert ist. Infolgedessen lässt sich das Arbeiten mit authentischen Texten empfehlen, und zwar schon am Anfang des ersten akademischen Jahres. Hierfür bietet die textgrammatische Methode einen guten Ansatzpunkt, sowohl hinsichtlich deduktiven als auch induktiven didaktischen Vorgehensweisen. Als Grundstrukturen kommen die folgenden in Frage: Verbalklammer, Nominalklammer und Adjunktklammer; Präpositionalphrase; Satzfelder (Vorfeld, Mittelfeld, Nachfeld); Verbzweitstellung; Topikalisierung der Felder; Satzglieder; Verbvalenz; Leerstellen.

- In der zweiten Erwerbsphase beginnen die Lerner die spezifischen Strukturen der L2 bewusst zu bearbeiten bzw. Ähnlichkeiten und Unterschiede zur L1 zu analysieren. In der didaktischen Praxis ist hier eine kontrastive Vorgangsweise möglich, indem die spezifischen Strukturen der deutschen Sprache - Verbalklammer, Verbzweitstellung und Vorfeldbesetzung - mit jenen des Italienischen verglichen werden. Dieser Vergleich zwischen den beiden Sprachen kann von den folgenden Betrachtungen ausgehen: Die deutsche Sprache besitzt eine zweiteilige Verbalstruktur (Verbalklammer, VK), die es ermöglicht, den Deklarativsatz in Satzfelder zu unterteilen, wobei sich Vorfeld (VF), Mittelfeld (MF) und Nachfeld (NF) unterscheiden lassen. Bei der Beschreibung ist das Augenmerk insbesondere auf die Stellung des Vorverbs (VV) innerhalb des deutschen Satzes zu richten, ein Phänomen, das als Hauptmerkmal des Deutschen betrachtet werden kann: Dadurch unterscheidet sich die syntaktische Ordnung der beiden Sprachen. Bei der kontrastiven Beschreibung sollte unterstrichen werden, dass sich im Italienischen keine Klammerstruktur nachweisen lässt: Im Hauptsatz ist ein konjugiertes Verb vorhanden, das ermöglicht, eine präverbale und eine postverbale Stellung zu unterscheiden. $\mathrm{Zu}$ diesem Punkt können die typischen Strukturen der zwei Sprachen miteinander verglichen werden: Für die deutsche Sprache betont man die Rolle der Verbalklammer und der durch das Vorverb eingenommenen Stellung; für die italienische Sprache die syntaktisch-semantische Kontinuität des Kerns Subjekt-konjugiertes Verb; die deutsche Vorfeldbesetzung kann mit

\footnotetext{
${ }^{22}$ Für eine detaillierte Beschreibung der Gestaltung des italienischen universitären DaF-Unterrichts vgl. Foschi Albert 2007.

${ }^{23}$ In den nachfolgenden Überlegungen wird die vierte Phase der Konsolidierung nicht betrachtet, da es sich dabei - wie schon erwähnt - eher um eine "Idealphase" handelt, in der der Lernende alle Regeln des deutschen Satzbaus beherrscht. Diese Phase wird effektiv nur durch eine begrenzte Anzahl von Studierenden erreicht.
} 
der Besetzung der italienischen präverbalen Stellung verglichen werden, indem man die Typologie und Quantität der Satzglieder beschreibt, die in den beiden Fällen auftreten. Diese Beschreibung kann sich auf typische Phänomene in den beiden Sprachen konzentrieren. In Bezug auf das Deutsche: das Auftreten eines einzigen Satzgliedes im VF und die Möglichkeit einer XVS-Ordnung; in Bezug auf das Italienische: das Pro-drop-Phänomen und die Möglichkeit der Nullbesetzung der präverbalen Position oder ihre Besetzung durch mehrere Satzglieder. Die Verwendung der Ausgangssprache als Blickperspektive könnte auch bei der Beschreibung anderer L2-spezifischer Strukturen nützlich sein.

- In der letzten Erwerbsphase, in der sich die Lerner bei der Bearbeitung der Zielstrukturen eher von der L1 distanzieren können und Verwechslungen öfter als die Interferenzen aus der L1 vorkommen, kann sich die Beschreibung der deutschen Grammatik vor allem auf bestimmte Strukturen der L2 konzentrieren. Dabei kann der Vergleich innerhalb der Zielsprache erfolgen, und zwar zwischen Strukturen der Zielsprache, die ähnlich sind und intralinguale Interferenzen verursachen können. Die Ergebnisse bestätigen, dass Deklarativsätze mit XVS-Ordnung oft mit Nebensätzen mit Verbendstellung verwechselt werden. Die beiden Strukturen können sowohl theoretisch als auch praktisch durch Textanalyse und Textproduktion miteinander verglichen werden.

Eine solche didaktische Praxis braucht adäquate didaktische Materialien, d. h. grammatische Beschreibungen des Deutschen, die auf Forschungsergebnissen basieren und Lernmechanismen stimulieren, die zur Erreichung von Sprachbewusstheit beitragen, d. h. sprachliche Vorkenntnisse aus der L1 und erworbene L2-Kenntnisse durch Assoziation aktivieren. Zurzeit haben die theoretischen Kurse der Lingua e Traduzione - Lingua Tedesca (L-LIN/14) an den italienischen Universitäten üblicherweise theoretische Aspekte der deutschen Linguistik bzw. Grammatik als Lerngegenstand ${ }^{24}$. Als didaktische Materialien werden dabei vor allem Einführungen in die theoretische Linguistik oder in die Geschichte bzw. Grammatik der deutschen Sprache benutzt, welche oft nicht kontrastiv angelegt sind (vgl. z. B. dazu WöllsteinLeistein 1997, Bosco Coletsos ${ }^{2} 2003$ ). In den letzten Jahren gibt es aber auch Versuche, didaktische Beschreibungen des Deutschen zu verfassen, die spezifisch auf italophone Studierende ausgerichtet sind (Tommaselli 2003, Di Meola 2004, Bosco Coletsos 1997, Blühdorn/ Foschi Albert 2006, Foschi Albert 2008)25. Es existiert aber noch keine vollständige kontrastive Grammatik Deutsch-Italienisch, die das Kontrastivitätsprinzip und die besonderen Merkmale des DaF-Erwerbsprozesses bei italophonen Studierenden berücksichtigt.

\section{Literatur}

Ballestracci, Sabrina (2006): Zum DaF-Erwerb ausgewählter grammatischer Strukturen der deutschen Sprache bei italophonen Studierenden der Pisaner Facoltà di Lingue e Letterature Straniere. Diss. Pisa.

Ballestracci, Sabrina (2008): "Überindividuelle Merkmale des Grammatikerwerbs im Unterricht des Deutschen als Fremdsprache durch italophone Studierende. Ergebnisse einer empirischen Untersuchung". Deutsch als Fremsprache 45/3: 160-169.

Blühdorn, Hardarik/Foschi Albert, Marina (2006): Lettura e comprensione del testo in lingua tedesca. Strategie inferenziali e grammaticali, tecniche euristiche, materiale illustrativo. Pisa.

\footnotetext{
${ }^{24}$ Die von Foschi Albert vorgenommene Untersuchung stellt fest, dass die Kurse für Lingua e Traduzione - Lingua Tedesca an den italienischen Universitäten vor allem die folgenden Themen zum Gegenstand haben: Geschichte der deutschen Sprache; Beschreibung der deutschen Sprachen in synchroner und diachroner Sicht; Kontrastive Linguistik Deutsch-Italienisch; Einführung in die deutsche Linguistik (vgl. Foschi Albert 2007: 156-158).

${ }^{25}$ Nach der statistischen Untersuchung von Foschi Albert beschäftigt sich 54\% (26:48) der universitären Kurse in Italien mit der kontrastiven Beschreibung Deutsch-Italienisch (vgl. Foschi Albert 2007: 156f.).
} 
Bosco Coletsos, Sandra (1997): Tedesco e italiano: un confronto: appunti morfo-sintattici, lessicali e fonetici. Alessandria.

Bosco Coletsos, Sandra $\left(2003^{2}\right)$ : Storia della lingua tedesca. Torino.

Catalani, Luigi (1993): Die Stellung der Satzelemente im deutschen und im Italienischen. Frankfurt am Main.

Clahsen, Harald (1980): "Psycholinguistic Aspects of L2 Acquisition". In: Felix, Sascha W. (ed.): Second Language Development: Trends and Issues. Tübingen: 57-79.

Clahsen, Harald (1982): Spracherwerb in der Kindheit. Eine Untersuchung zur Entwicklung der Syntax bei Kleinkindern. Tübingen.

Clahsen, Harald/Meisel, Jürgen/Pienemann, Manfred (1983): Deutsch als Zweitsprache. Der Spracherwerb ausländischer Arbeiter. Tübingen.

Crespi Günther, Marina (1998): Frasi complesse in tedesco L2. Percorsi di apprendimento della sintassi della subordinazione. Milano.

Diehl, Erika et al. (2000): Grammatikunterricht: Alles für der Katz? Untersuchungen zum Zweitsprachenerwerb Deutsch. Tübingen.

Di Meola, Claudio (2004): La linguistica tedesca. Un'introduzione con esercizi e bibliografia ragionata. Roma.

Drach, Erich (1937): Grundgedanken der deutschen Satzlehre. Frankfurt am Main.

Dudenredaktion (20057): Die Grammatik. Mannheim et al. (= Der Duden in 12 Bänden 4).

Dulay, Heidi/Burt, Marina/Krashen, Stephen (1982): Language Two. New York.

Dürscheid, Christa $\left(2007^{4}\right)$ : Syntax. Grundlagen und Theorien. Göttingen.

Edmondson, Willis/House, Juliane (2000): Einführung in die Sprachlehrforschung. Tübingen/ Basel.

Eisenberg, Peter (1999): Grundriss der deutschen Grammatik. Band 2: Der Satz. Stuttgart.

Foschi Albert, Marina (2007): "Gli insegnamenti di Lingua e Traduzione - Lingua Tedesca (L-LIN/14) nelle università italiane. Un sondaggio". daf-Werkstatt 9/10. Arezzo: 149-164.

Foschi Albert, Marina (2008): Elementi base di grammatica tedesca. Costituenti sintattici e occupazione dei campi. Con note di carattere contrastivo (tedesco-italiano). Pisa.

Gallmann, Peter/Sitta, Horst (im Druck): Sprachen im Vergleich. Deutsch - Italienisch Ladinisch. Der zusammengesetzte Satz. Bozen.

Klein Gunnewiek, Lisanne (2000): Sequenzen und Konsequenzen. Zur Entwicklung niederländischer Lerner im Deutschen als Fremdsprache. Amsterdam.

Menzel, Barbara (2004): Genuszuweisung im DaF-Erwerb. Psycholinguistische Prozesse und didaktische Implikationen. Berlin.

Pienemann, Manfred (1998): Language Processing and Second Language Development. Processability Theory. Amsterdam.

Terrasi Haufe, Elisabetta (2004): Der Schulerwerb von Deutsch als Fremdsprache. Eine empirische Untersuchung am Beispiel der italienischsprachigen Schweiz. Tübingen.

Tomaselli, Alessandra (2003): Introduzione alla sintassi del tedesco. Bari.

Thomoglou, Polichrona (2004): Genuserwerb bei griechischen Lernern des Deutschen. Eine empirische Untersuchung und didaktische Implikationen. Frankfurt am Main.

Tschirner, Erwin (1999): "Lernergrammatiken und Grammatikprogression". In: Skibitzki, Bernd/Wotjak, Barbara (eds.): Linguistik und Deutsch als Fremdsprache. Festschrift für Gerhard Helbig zum 70. Geburtstag. Tübingen: 227-239.

Weinrich, Harald (1993): Textgrammatik der deutschen Sprache. Mannheim et al.

Wöllstein-Leisten et al. (1997): Deutsche Satzstruktur. Grundlagen der syntaktischen Analyse. Tübingen.

Zifonun, Gisela et al. (1997): Grammatik der deutschen Sprache. Berlin. 